\title{
Synthesis of super high molecular weight copolymer of AM/NaA/AMPS by oxidation-reduction and controlled radical polymerization
}

\author{
Ji Yan-feng ${ }^{1} \cdot$ Cao Xu-long ${ }^{1} \cdot$ Zhu Yang-wen $^{1} \cdot$ Xu Hui $^{1} \cdot$ Sun Xiu-zhi ${ }^{1} \cdot$ Li Hai-tao $^{1}$
}

Received: 5 May 2019 / Published online: 14 November 2019

(C) The Author(s) 2019

\begin{abstract}
Super high molecular weight copolymers of AM/NaA/AMPS were prepared by oxidation-reduction [OR-P(AM/NaA/ AMPS)] and controlled radical polymerization [CR-P(AM/NaA/AMPS)]. The resulting copolymers were fully characterized, and the reaction conditions for their preparation were optimized. OR-P(AM/NaA/AMPS), CR-P(AM/NaA/AMPS), and conventional partially hydrolyzed polyacrylamide (HPAM) in brine solution were comprehensively characterized by thermogravimetric analysis, scanning electron microscopy, atomic force microscopy, and dynamic light scattering. OR$\mathrm{P}(\mathrm{AM} / \mathrm{NaA} / \mathrm{AMPS})$ and $\mathrm{CR}-\mathrm{P}(\mathrm{AM} / \mathrm{NaA} / \mathrm{AMPS})$ containing AMPS monomer showed better salt resistance, temperature tolerance, and viscosification property than the conventional HPAM polymer, making them more promising for enhanced oil recovery. Through comprehensive comparison and analysis, it was found that OR-P(AM/NaA/AMPS) was more conducive for high-temperature condition due to the existence of xanthone in OR-P(AM/NaA/AMPS). On the other hand, CR-P(AM/ NaA/AMPS) was more suitable for high-mineral atmosphere, which could be attributed to its higher intrinsic viscosity.
\end{abstract}

Keywords Oxidation-reduction polymerization - Controlled radical polymerization · Elemental composition · Thermogravimetry $\cdot$ Microcosmic aggregation morphology

\section{List of symbols}

$k_{\mathrm{B}} \quad$ The Boltzmann constant

$T \quad$ The absolute temperature, ${ }^{\circ} \mathrm{C}$

$R_{\mathrm{h} \text {,app }}$ The hydrodynamic radius of particle, $\mathrm{m}$

$D_{\text {app }} \quad$ The Doppler frequency shift, $\mathrm{Hz}$

\section{Greek letter \\ $\eta \quad$ The solvent viscosity, $\mathrm{mPa} \mathrm{s}$}

\section{Introduction}

Polyacrylamide (PAM) is an important type of hydro-soluble polymer, with flocculating, thickening, drag reduction, and dispersing characteristics. PAM has been used in various areas, ranging from coal and mineral processing, papermaking, water purification, drag reduction, to oil production

\section{Edited by Xiu-Qiu Peng}

Ji Yan-feng

jiyanfeng_001@163.com

1 Exploration and Development Research Institute, Shengli Oilfield, Dongying 257000, Shandong Province, People's Republic of China processes including oil well drilling, reservoir stimulation, water shutoff, profile modification, and particularly tertiary oil recovery. Partially hydrolyzed polyacrylamide (HPAM) is one of the most widely used water-soluble polymers for enhanced oil recovery (EOR) (Thomas 2007; Wever et al. 2011; Bao et al. 2010). In polymer flooding process, HPAM is used to thicken the injected water to mobilize capillary trapped water-flooded oil in the secondary stage, which improves the sweep efficiency to increase the oil recovery factor. However, due to shifting of oil production toward deeper, high-temperature and high-salinity (HTHS) reservoirs, conventional HPAM cannot be used to viscosify the displacing fluid under the HTHS conditions. This is because its viscosity decreases significantly under HTHS conditions due to a charge shielding effect (Gao 2013; Ladaviere et al. 1999). To overcome the limitations of HPAM, a great deal of research has been undertaken on the synthesis of thermally stable and salt-tolerant water-soluble polymers (Hong et al. 2005; Zhang et al. 2007; Feng et al. 2001; Sarsenbekuly et al. 2017).

The methods for developing improved PAMs are mainly as follows (Corlay et al. 1996; Wu et al. 2001; Zhong et al. 2010; Guo et al. 2014). Firstly, the synthesis of non-associative polymers with structural monomers having temperature stability and salt tolerance properties, and introducing 
functional structural monomers, such as 2-acrylamide2-methylpropanesulfonic acid (AMPS), N-vinylpyrrolidone (NVP), and N-vinylamides (NVAs) with desirable properties including restricted hydrolysis, complexing high valence cations, great hydration capacity, and enhancing the rigidity of high molecular chain (Ding et al. 2014; Song et al. 2007; Ma et al. 2002; Liu et al. 2013). Secondly, the synthesis of polymers with special interactions, such as hydrophobic associated polymer, amphoteric ionic polymer, and colloidal dispersion gel (He et al. 2014; Mao et al. 2015). Thirdly, the synthesis of light cross-linked polymers with increased difficulty in conformational transition, high salt tolerance and increased viscosity due to the presence of cross-linked structure (Bara et al. 2008; Kafouris et al. 2006; Yang et al. 2003). Fourthly, the synthesis of super high molecular weight polymers, which can enhance the thickening capacity by increasing the hydrodynamic volume of the solution of high molecular weight polymer (Ye et al. 2015). Among them, the polymer obtained by the second and third methods is prone to a large number of insoluble substances, so the first and fourth are the main methods for our study.

In this study, two super high molecular weight copolymers of acrylamide (AM)/sodium acrylate (NaA)/AMPS were prepared by oxidation-reduction (OR) and controlled radical (CR) polymerization, respectively. In the controlled radical (CR) polymerization system, the balance between increasing free radicals and various dormant species is used to control the molecular weight, molecular weight distribution, and terminal functional free radical polymerization reaction of the polymer. Oxidation-reduction (OR) polymerization refers to the polymerization of olefinic monomers initiated by free radicals generated by redox reactions. Polymers synthesized by different methods may exhibit different properties, taking this into account, this paper discusses the effects of AMPS on the molecular structure, stretching property and microcosmic morphology of high molecular weight polymers prepared by different methods to provide theoretical and methodological support for the preparation of acrylamide-based polymers.

\section{Materials and methods}

\subsection{Materials}

Acrylamide (AM, 99.5\%, Changjiu Agri-Scientific Co. Ltd, Nanchang, China), 2-acrylamide-2-methylpropanesulfonic acid (AMPS, 99.2\%, Shandong Lianmeng Chemical Co. Ltd, China), and macroinitiator polyacrylamide-xanthone (PAM-XAN) were prepared in our laboratory using known procedures. Sodium hydroxide, ethylene diamine tetraacetic acid (EDTA), low temperature OR initiator (potassium persulfate and sodium hydrogen sulfite), azo initiator (azodiisobutyronitrile), and other solvents were all analytical grade and purchased from Aladdin Chemical Reagent Factory (Shanghai, China). The water used in this study was double distilled using an all-glass apparatus, and nitrogen with a purity of $99.99 \%$ was used. low temperature OR initiator, Azo initiator.

\subsection{Synthesis of copolymers}

\subsubsection{Synthesis of oxidation-reduction polymer}

The terpolymer OR-P (AM/NaA/AMPS) was prepared by the oxidation-reduction copolymerization of AM, sodium acrylate ( $\mathrm{NaA})$, and AMPS in pure water, and the $\mathrm{pH}$ was adjusted around 9 using $\mathrm{NaOH}$. After 30 min of $\mathrm{N}_{2}$ purge, a certain amount of initiator was injected into the solution. The polymerization was carried out at $10{ }^{\circ} \mathrm{C}$ under $\mathrm{N}_{2}$ atmosphere for $4 \mathrm{~h}$, and the resulting product was obtained by adding a certain amount of sodium hydroxide into the reaction mixture, followed by freeze-drying, affording the final terpolymer product as a white powder.

The effects of different types of initiator on heating rate, intrinsic viscosity, and apparent viscosity were investigated; the effects of AMPS content were also studied.

\subsubsection{Synthesis of macroinitiator polyacrylamide-xanthone}

Adding $4 \mathrm{~g}$ acrylamide monomers into three flasks of $100 \mathrm{~mL}$, with $50 \mathrm{ml} \mathrm{N}, \mathrm{N}$-dimethylformamide dissolving the monomers, then $0.4 \mathrm{~g}$ bi-xanthene was added to stir and deaerate with nitrogen for $40 \mathrm{~min}$, the reaction was maintained in a water bath of $50{ }^{\circ} \mathrm{C}$ with stirring and aeration. After $2 \mathrm{~h}$, white precipitation appeared and the reaction stopped after $6 \mathrm{~h}$. The reaction liquid was centrifuged in a centrifuge at $4000 \mathrm{rad} / \mathrm{min}$ speed for $20 \mathrm{~min}$. The white precipitate was washed in acetone and centrifuged. The precipitate was dried in a vacuum oven and stored in a refrigerator.

\subsubsection{Synthesis of controlled radical polymer}

The terpolymer CR-P(AM/NaA/AMPS) was prepared by controlled radical polymerization of AM and AMPS in pure water, and the $\mathrm{pH}$ was adjusted around 9 using $\mathrm{NaOH}$ followed by 30 min of $\mathrm{N}_{2}$ purge. Then, a certain amount of macroinitiator (PAM-XAN) was injected into the solution. The polymerization was carried out at $40{ }^{\circ} \mathrm{C}$ under $\mathrm{N}_{2}$ atmosphere for $6 \mathrm{~h}$. The resulting product was obtained by adding the reaction mixture into a certain amount of sodium hydroxide, followed by freeze-drying, affording the final terpolymer product as a white solid. The synthetic route is shown in Fig. 1. 

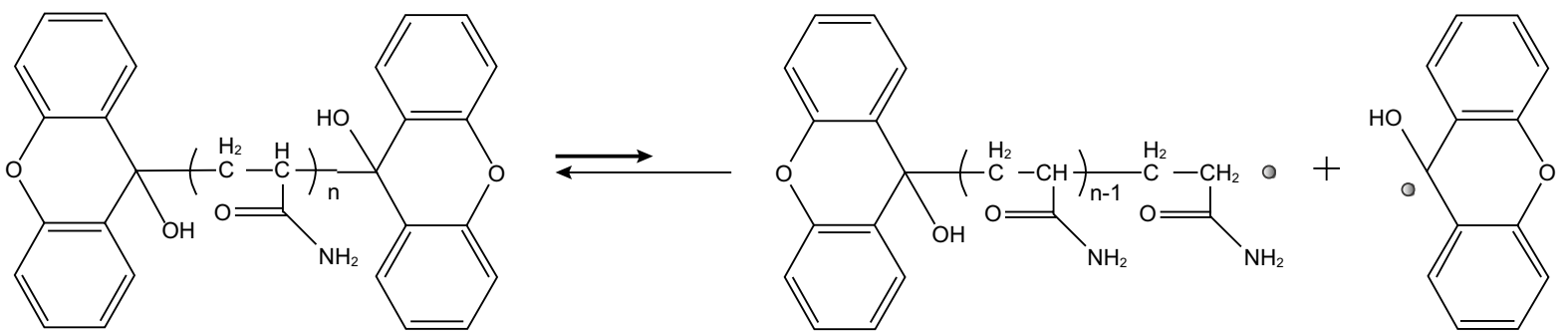

(PAM-XAN)<smiles>C=CC(=O)NC(C)(C)CS(=O)(=O)O</smiles>

(AMPS)<smiles>C=CC(=O)O</smiles>

(AM)

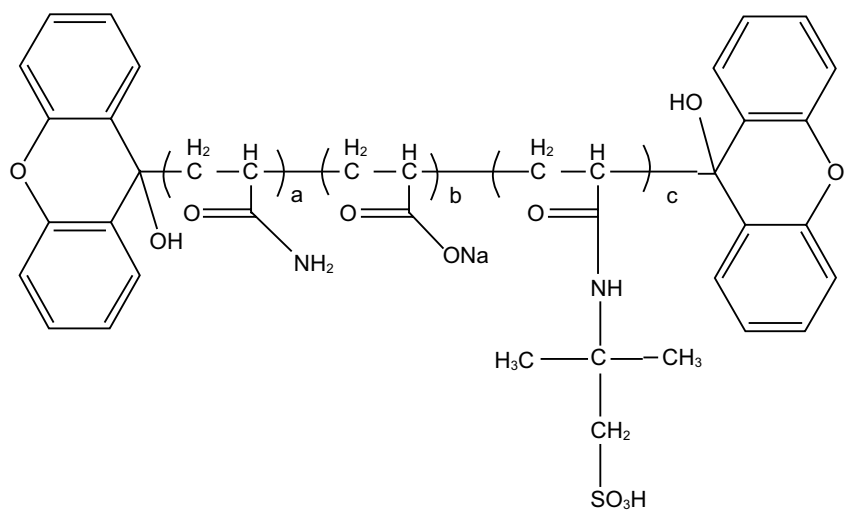

CR-P(AM/NaA/AMPS)

Fig. 1 Synthesis process of CR-P(AM/NaA/AMPS)

\subsection{Characterizations}

\subsubsection{Infrared spectroscopy}

The IR spectra of the samples were measured with $\mathrm{KBr}$ pellets using a WQF-520A IR Spectrophotometer (Beijing Rayleigh Analytical Instrument Company) in the optical range, $4500-400 \mathrm{~cm}^{-1}$, by averaging 32 scans at a resolution of $4 \mathrm{~cm}^{-1}$.

\subsubsection{Elementary analysis}

The elementary analysis of the AM/AA/NSFM copolymer was carried out using a Vario EL-III elemental analyzer. The content of different element in the copolymer can be obtained by detecting the gases, which are the decomposition products of the copolymer at high temperature.

\subsubsection{Thermogravimetric analysis}

The thermal weight loss curves of polymer samples were measured using a TGA-Q500 type thermal analyzer, and the structural changes of the polymers were evaluated according to the thermogravimetric curves. The test conditions of TGA were as follows: oxygen atmosphere at a flow rate of $100 \mathrm{~mL} / \mathrm{min}$; heating rate of $10{ }^{\circ} \mathrm{C} / \mathrm{min}$; and test temperature range of $25-700{ }^{\circ} \mathrm{C}$.

\subsubsection{Dynamic light scattering}

DLS technique has been widely used for determining the hydrodynamic radius of polymers by testing the intensity fluctuation change of sample. The working principle is based on the variation in the intensity of scattered light, which is the diffusion coefficient of the molecule tested by Doppler 
frequency shift- $D_{a p p}$. Using Stokes-Einstein formula, the hydrodynamic radius of particle can be derived as follows:

$R_{\mathrm{h} \text {,app }}=k_{\mathrm{B}} T /\left(6 \pi \eta D_{\text {app }}\right)$

where $k_{\mathrm{B}}$ is the Boltzmann constant, $\eta$ is the solvent viscosity, and $T$ is the absolute temperature.

DLS spectrum was obtained using a Laser Light Scattering Spectrometer (ALV-5000/E/WIN Multiple Tau Digital Correlator). The sample was centrifuged to remove dust before analysis, and the test temperature was $40{ }^{\circ} \mathrm{C}$.

\subsubsection{Scanning electronic microscopy}

SEM images were taken using a JMS-6380LV scanning electron microscope. The polymer solution was frozen by liquid nitrogen and then was made electrically conductive by coating a thin layer of gold in vacuum (approximately $300 \AA$ ) at $30 \mathrm{~W}$ for $30 \mathrm{~s}$. The pictures were taken at an excitation voltage of $10 \mathrm{kV}$ and a magnification of $5000 \times$.

\subsubsection{Atomic force microscopy}

Atomic force microscopy was used to investigate the surface morphology and thickness of HPAM and the two copolymers. AFM images were taken using a commercial Nanoscope III (Digital Instruments, Santa Barbara, CA) using a $\mathrm{Si}_{3} \mathrm{~N}_{4}$ probe to analyze the apparent morphology of polymer $(1000 \mathrm{mg} / \mathrm{L})$.

\section{Results and discussion}

\subsection{Optimization of oxidation-reduction polymerization}

The experimental results in Table 1 show that the intrinsic and apparent viscosities of OR-P(AM/NaA/AMPS) by using the complex initiation system of OR initiator, azo initiator, and additive were greater than that by using either OR initiator or azo initiator alone, while the heating rate of

Table 1 Effect of initiator systems on the molecular weight and viscosity of the polymer

\begin{tabular}{lllc}
\hline Initiator systems & $\begin{array}{l}\text { Heating } \\
\text { rate, }{ }^{\circ} \mathrm{C} / \mathrm{h}\end{array}$ & $\begin{array}{l}\text { Intrinsic } \\
\text { viscosity, } \\
\mathrm{mL} / \mathrm{g}\end{array}$ & $\begin{array}{l}\text { Apparent } \\
\text { viscosity, } \\
\mathrm{mPa} \mathrm{s}\end{array}$ \\
\hline $\begin{array}{l}\text { Oxidation-reduction initiator } \\
\text { Azo initiator }\end{array}$ & 30.0 & 2100 & 7.8 \\
$\begin{array}{l}\text { Oxidation-reduction initiator, } \\
\text { Azo initiator }\end{array}$ & 24.5 & 2200 & 7.8 \\
$\begin{array}{l}\text { Oxidation-reduction initiator, } \\
\text { Azo initiator, assistant agent }\end{array}$ & 8.0 & 3350 & 11.2 \\
\hline
\end{tabular}

Table 2 Effect of functional monomer AMPS content on the viscosity of polymer

\begin{tabular}{lll}
\hline Sample & AMPS content, $\%$ & $\begin{array}{l}\text { Apparent } \\
\text { viscosity, } \\
\text { mPa s }\end{array}$ \\
\hline $1 \#$ & 20.0 & 17.9 \\
$2 \#$ & 15.0 & 15.3 \\
$3 \#$ & 12.0 & 15.1 \\
$4 \#$ & 10.0 & 14.2 \\
\hline
\end{tabular}

polymerization system was quite different under complex initiation systems. Thus, it can be supposed that the complex initiation system of OR initiator, azo initiator, and additive can regulate the decomposition rate of initiator, making the polymerization reaction more stable. Therefore, low temperature OR initiator, azo initiator, and additive were used in this study.

Table 2 shows that the apparent viscosity increased with increasing AMPS content. However, AMPS is far more expensive than AM. Therefore, the final AMPS content was chosen to be around $12 \%-15 \%$.

\subsection{Optimization of controlled radical polymer}

The intrinsic viscosity of the controlled free radical copolymer products with different AMPS contents was investigated at different temperatures.

Table 3 Intrinsic viscosity of $\mathrm{CR}-\mathrm{P}(\mathrm{AM} / \mathrm{NaA} / \mathrm{AMPS})$ is prepared under different temperature conditions

\begin{tabular}{lllll}
\hline $\begin{array}{l}\text { Mac- } \\
\text { roinitia- } \\
\text { tor, mg }\end{array}$ & AM, & AMPS, g & $\begin{array}{l}\text { Reaction } \\
\text { temperature, } \\
{ }^{\circ} \mathrm{C}\end{array}$ & Intrinsic viscosity, mL/g \\
\hline 30 & 1.8 & 0.2 & 55 & 1873 \\
30 & 1.8 & 0.2 & 50 & 1976 \\
30 & 1.8 & 0.2 & 45 & Cross-linked \\
30 & 1.8 & 0.2 & 40 & Cross-linked \\
30 & 1.6 & 0.4 & 55 & 1632 \\
30 & 1.6 & 0.4 & 50 & 1818 \\
30 & 1.6 & 0.4 & 45 & 1877 \\
30 & 1.6 & 0.4 & 40 & 2065 \\
30 & 1.4 & 0.6 & 55 & 1223 \\
30 & 1.4 & 0.6 & 50 & 1469 \\
30 & 1.4 & 0.6 & 45 & 1755 \\
30 & 1.4 & 0.6 & 40 & 1826 \\
30 & 1.2 & 0.8 & 55 & 997 \\
30 & 1.2 & 0.8 & 50 & 1252 \\
30 & 1.2 & 0.8 & 45 & 1516 \\
30 & 1.2 & 0.8 & 40 & 1644 \\
\hline
\end{tabular}


The experimental results in Table 3 show that at identical monomer concentrations, the intrinsic viscosity of CR-P(AM/NaA/AMPS) increased gradually with decreasing polymerization temperature, indicating that the polymerization reaction was more stable at low temperature. In particular, when the proportion of AM/AMPS (mass ratio 9:1) and the polymerization temperature were low, crosslinking reactions were more likely to occur in CR-P(AM/ NaA/AMPS), decreasing the solubility. The results showed that the cross-linking reaction was effectively suppressed with increasing AMPS content (AM/AMPS mass ratio changed to $8: 2,7: 3$ and $6: 4$ ). However, with increasing AMPS content, the intrinsic viscosity of the prepared polymers significantly reduced. Based on the cross-linking reactions and the intrinsic viscosity, the optimum values of mass ratio of AM/AMPS and reaction temperature were 8:2 and $40^{\circ} \mathrm{C}$, respectively.

Table 4 illustrates the changes in the intrinsic viscosity with different amounts of initiator and concentration of monomer at a fixed AM/AMPS mass ratio of 8:2. Table 4 shows that the intrinsic viscosity of copolymer increased with decreasing concentration of macroinitiator. With increasing monomer percentage (from 25 to $35 \%$ ), the intrinsic viscosity increased first and then decreased. The optimum dosage of macroinitiator and critical monomer content was selected as follows to obtain the maximum intrinsic viscosity: macroinitiator dosage of $10 \mathrm{mg}$ and monomer content of $30 \%$.

Table 4 Intrinsic viscosity of CR-P(AM/NaA/AMPS) prepared under different dosages of initiator and monomer content

\begin{tabular}{lll}
\hline Macroinitiator, mg & Monomer content, $\%$ & $\begin{array}{l}\text { Intrinsic } \\
\text { viscosity, } \\
\mathrm{mL} / \mathrm{g}\end{array}$ \\
\hline 30 & 25 & 2065 \\
25 & 25 & 2182 \\
20 & 25 & 2247 \\
15 & 25 & 2386 \\
10 & 25 & 2518 \\
30 & 30 & 2211 \\
25 & 30 & 2407 \\
20 & 30 & 2596 \\
15 & 30 & 2681 \\
10 & 30 & 2772 \\
30 & 35 & 2170 \\
25 & 35 & 2235 \\
20 & 35 & 2372 \\
15 & 35 & 2470 \\
10 & 35 & 2520
\end{tabular}

\subsection{Molecular structure and elemental characterization}

The IR spectra are shown in Fig. 2. The IR spectra of OR$\mathrm{P}(\mathrm{AM} / \mathrm{NaA} / \mathrm{AMPS})$ indicated that monomer AMPS was copolymerized successfully onto the backbone of PAM chains, as confirmed by the peaks at 1194 and $1119 \mathrm{~cm}^{-1}$ in the IR spectrum of OR-P(AM/NaA/AMPS), which are the characteristic absorption peaks of sulfonic group due to the $\mathrm{S}=\mathrm{O}$ stretching vibration of $\mathrm{SO}_{3}{ }^{2-}$ in AMPS. In the IR spectrum of CR-P (AM/NaA/AMPS), the main peaks for the infrared vibration of acrylamide at $3438 \mathrm{~cm}^{-1}, 2929 \mathrm{~cm}^{-1}$, and $1633 \mathrm{~cm}^{-1}$, and characteristic peak of sulfonic acid at $1194 \mathrm{~cm}^{-1}$ were observed. In addition, the characteristic absorption peak of the benzene ring skeleton at $1451 \mathrm{~cm}^{-1}$ was also observed, indicating the existence of xanthone on the molecular skeleton. According to the IR spectrum of HPAM, the main infrared vibration peaks of acrylamide were observed at $3438 \mathrm{~cm}^{-1}, 2929 \mathrm{~cm}^{-1}$, and $1633 \mathrm{~cm}^{-1}$, but the infrared vibration peaks of sulfonate group or xanthone were not observed.

The different elemental contents in the copolymer and HPAM were obtained by detecting the gases, which are the

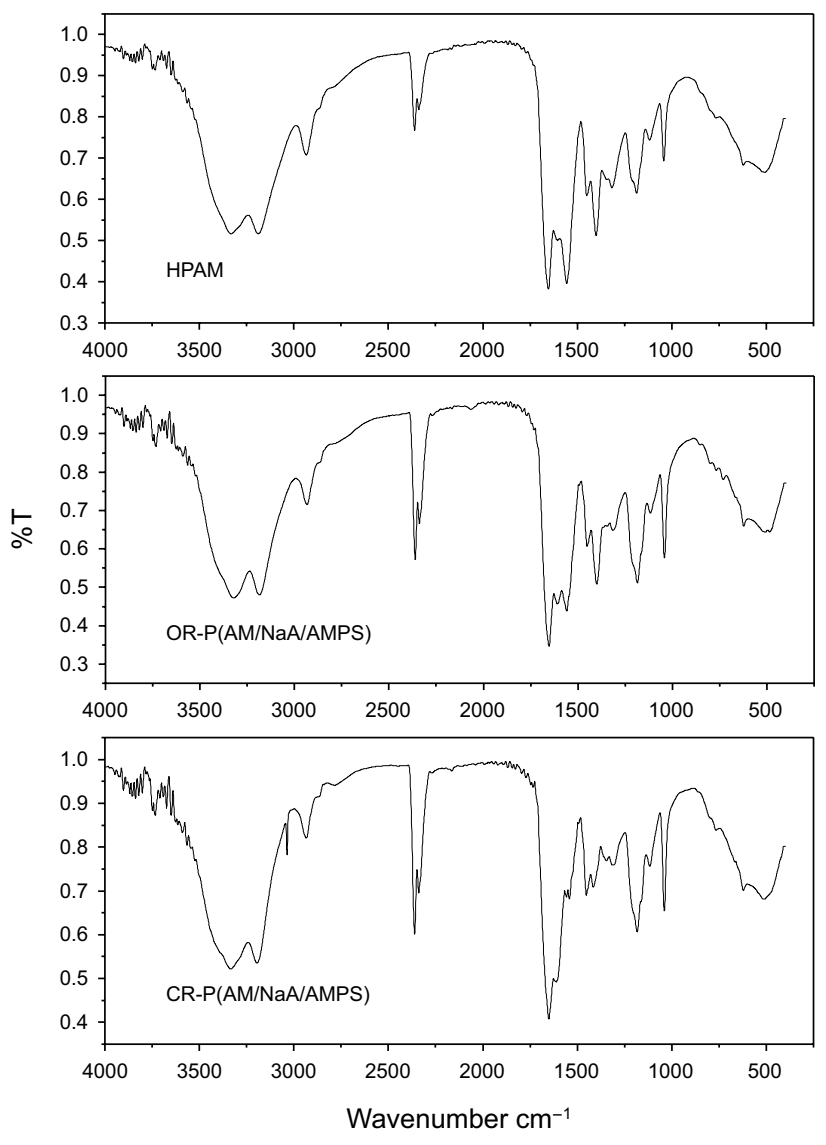

Fig. 2 Infrared spectrogram of polymers 
Table 5 Analysis and comparison of elements in copolymers and HPAM

\begin{tabular}{|c|c|c|c|c|c|}
\hline \multirow[t]{2}{*}{ Polymer } & \multicolumn{5}{|c|}{ Element } \\
\hline & $\mathrm{C}$ & $\mathrm{H}$ & $\mathrm{N}$ & $\mathrm{O}$ & S \\
\hline HPAM & 38.28 & 6.55 & 13.43 & 29.65 & \\
\hline OR-P & 39.73 & 6.59 & 12.51 & 30.42 & 1.04 \\
\hline CR-P & 38.92 & 6.62 & 13.83 & 28.93 & 2.01 \\
\hline
\end{tabular}

decomposition products of the copolymer at high temperature. The experimental results are listed in Table 5. The conventional HPAM does not show any significant presence of sulfur, as no AMPS monomer was introduced into HPAM. For the two copolymers, a considerable percentage of sulfur was observed, confirming that AMPS was copolymerized onto the backbone of PAM chains.

\subsection{Analysis and characterization of thermal weight loss of polymer}

The thermal weight loss and thermogravimetric curves of the two copolymers were investigated and compared to that of the conventional HPAM.

Figure 3 shows that the initial weight loss temperature of HPAM was about $204.18{ }^{\circ} \mathrm{C}$, while the initial weight loss temperatures of OR-P(AM/NaA/AMPS) and CR-P(AM/ $\mathrm{NaA} / \mathrm{AMPS}$ ) were approximately $312.17^{\circ} \mathrm{C}$ and $257.20^{\circ} \mathrm{C}$, respectively. Compared to HPAM, the TG/TGA data of the two copolymers not displayed significant weight losses until $400.33{ }^{\circ} \mathrm{C}$ and $331.44{ }^{\circ} \mathrm{C}$, indicating that the thermal stabilities of two copolymers were enhanced by the addition of AMPS monomer. Moreover, OR-P(AM/NaA/AMPS) showed better temperature resistance, probably because of higher intrinsic viscosity of OR-P(AM/NaA/AMPS).

\subsection{Microcosmic aggregation morphology of polymer solution}

The microcosmic aggregation morphologies of OR-P(AM/ NaA/AMPS), CR-P(AM/NaA/AMPS), and HPAM were characterized and analyzed by DLS, freeze-etching SEM, and AFM at room temperature.

\subsubsection{Study on the hydrodynamic radius of polymer}

The hydrodynamic radius of the polymer was investigated by DLS, and the results are shown in Fig. 4. The hydrodynamic radius of the three types of polymers increased with increasing concentration. However, the hydrodynamic radius of both copolymers was considerably higher than that of
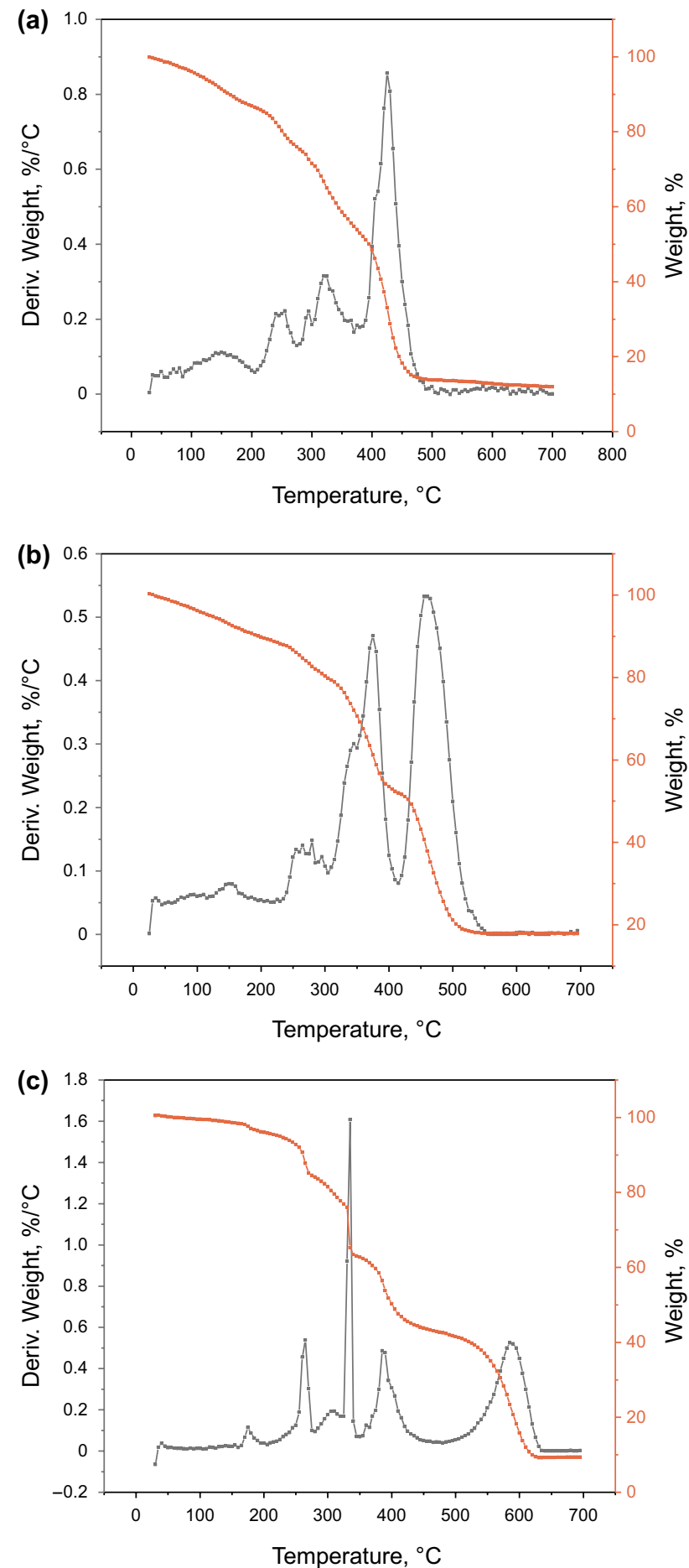

Fig. 3 TG curve of: a HPAM; b OR-P(AM/NaA/AMPS); c CR$\mathrm{P}(\mathrm{AM} / \mathrm{NaA} / \mathrm{AMPS})$

conventional HPAM, reflecting the better tackifying ability of the two copolymers. The average hydrodynamic radius of 
CR-P(AM/NaA/AMPS) was higher than that of OR-P(AM/ NaA/AMPS).

\subsubsection{Microstructure characterization of polymer solution by freeze-etching SEM}

The microstructures of two copolymers and HPAM in aqueous solution were investigated by freeze-etching SEM. The polymer solution was prepared in distilled water at a concentration of $0.1 \%$ and simulated water at a concentration of $0.2 \%$. The simulated water had a calcium ion content of $874 \mathrm{mg} / \mathrm{L}$. Under the same magnification conditions, the microstructures of the three types of polymer were observed and compared. The SEM images are shown in Figs. 5 and 6.

Figure 5 indicated the dense network structure of HPAM, OR-P(AM/NaA/AMPS), and CR-P(AM/NaA/AMPS) in distilled water, and the molecular chains in aqueous solution showed mutual aggregation and winding. Based on this effect, the friction between the molecular chains increased, and the polymer solution showed high viscosity.

As seen from Fig. 6, in the presence of high content of calcium and magnesium ions, the aggregation and entanglement of HPAM and copolymers solution decreased significantly, and the network structure formed in solution became sparser. This might be attributed to the existence of calcium ions, which can destroy the molecular aggregation and decrease the viscosity of polymer solution. However, the degree of damage to the network structure of the three polymer solutions was quite different. The molecular chains of OR-P(AM/NaA/AMPS) and CR$\mathrm{P}(\mathrm{AM} / \mathrm{NaA} / \mathrm{AMPS})$ still formed a network between the dense structure to a certain extent, while conventional HPAM solution showed more serious damage. Therefore, the apparent viscosity of copolymers was also higher. The

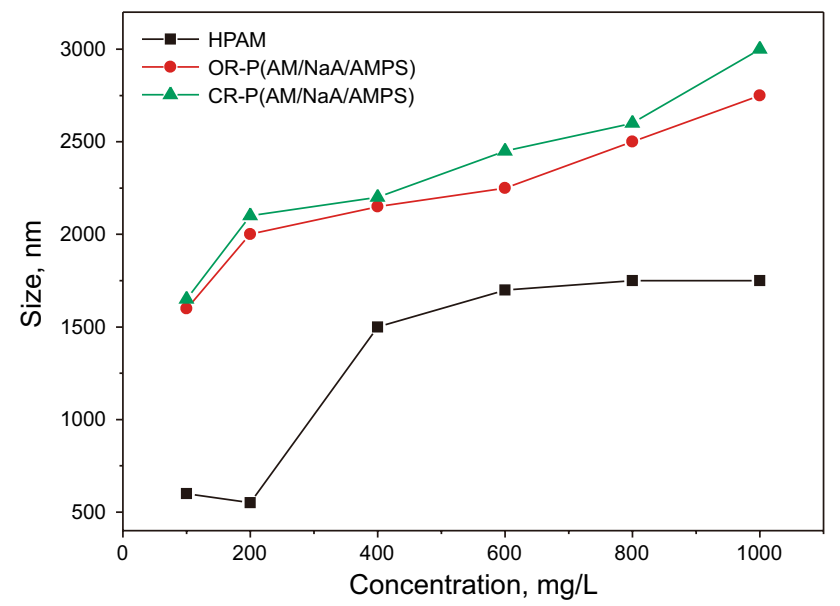

Fig. 4 Change of the average hydrodynamic radius of the polymer with the concentration
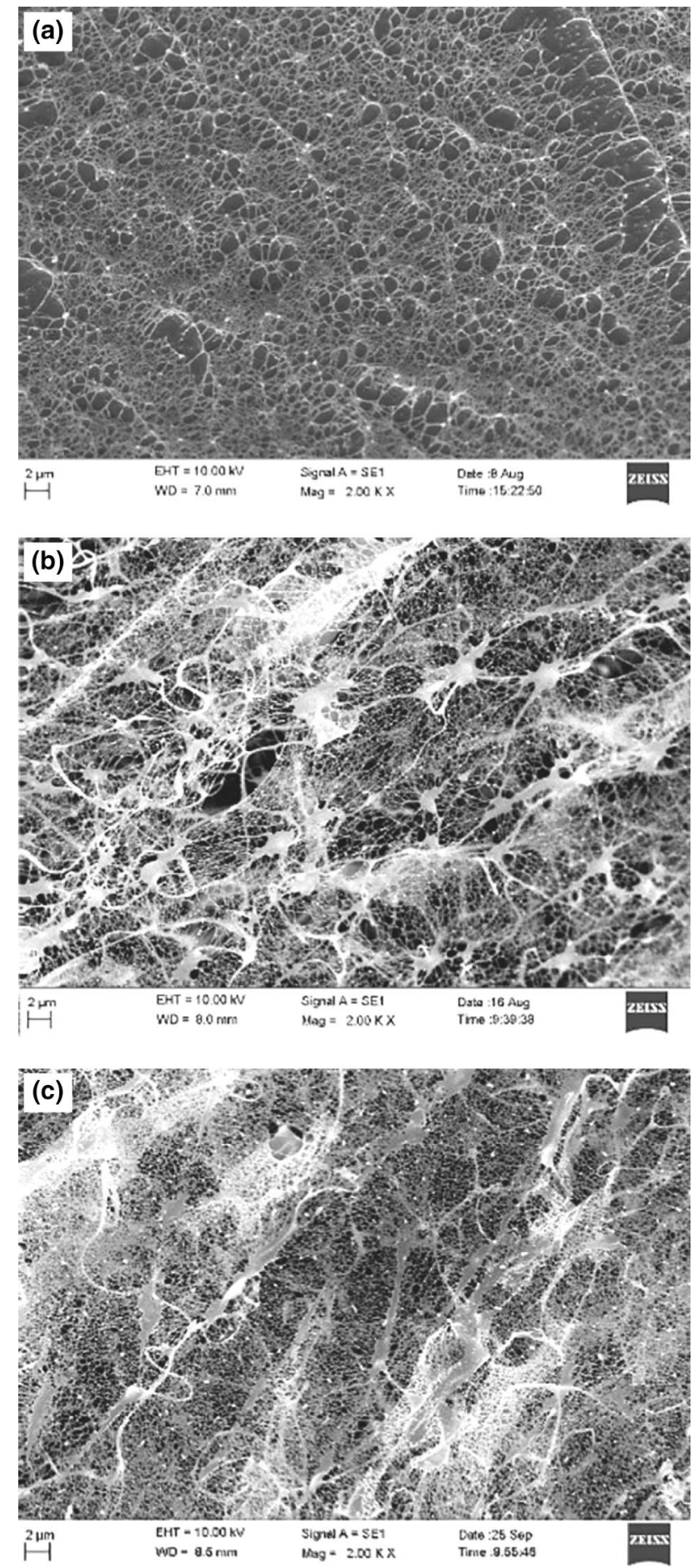

Fig. 5 Microaggregation morphology of $0.1 \%$ : a HPAM; b OR$\mathrm{P}(\mathrm{AM} / \mathrm{NaA} / \mathrm{AMPS})$; c CR-P(AM/NaA/AMPS)

network structure of CR-P(AM/NaA/AMPS) was more obvious than that of OR-P(AM/NaA/AMPS), resulting in increased salt tolerance (Gaynor et al. 1995; Save et al. 2007; Hideharu et al. 2010) which is consistent with the DLS result. 

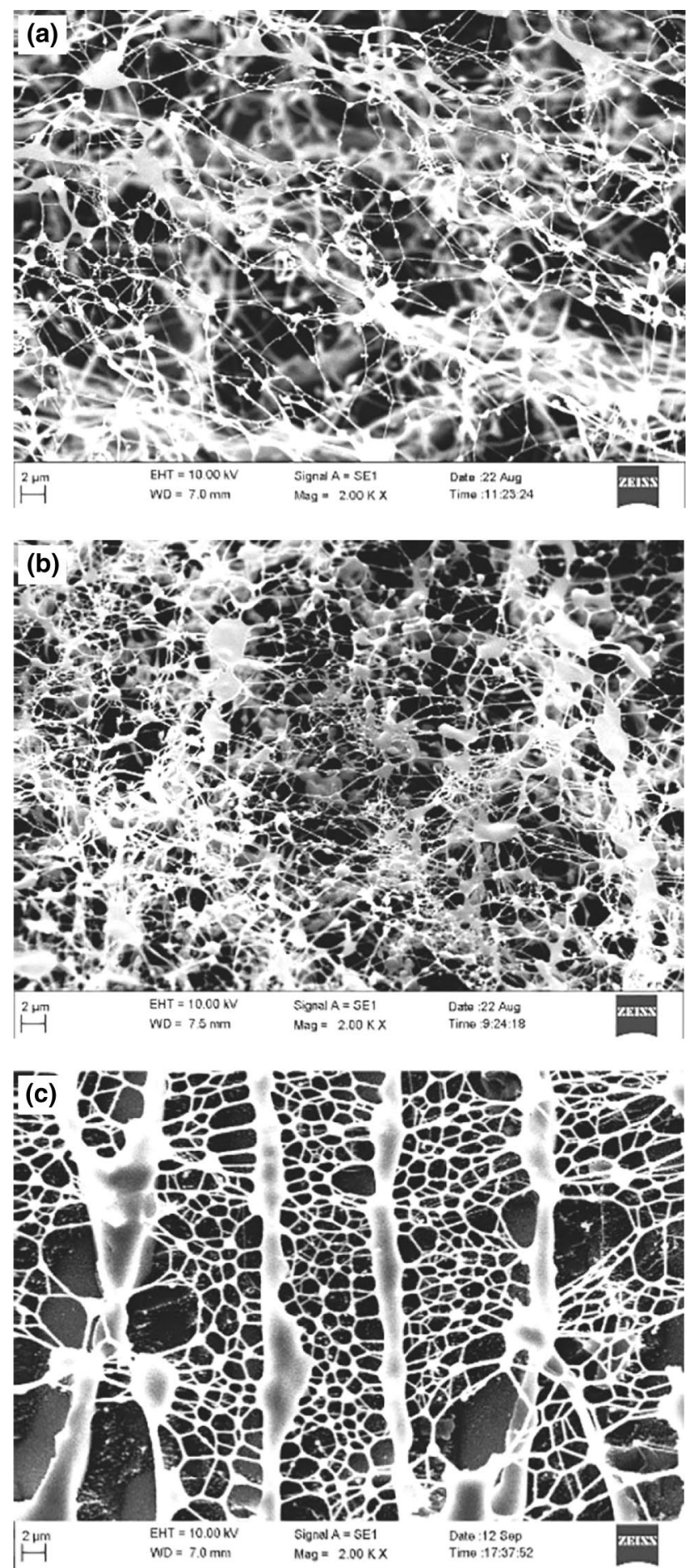

Fig. 6 Microaggregation morphology of $0.2 \%$ : a HPAM; b OR$\mathrm{P}(\mathrm{AM} / \mathrm{NaA} / \mathrm{AMPS})$; c CR-P(AM/NaA/AMPS)

\subsubsection{AFM characterization of the morphology of the polymer aggregates}

The micromorphology of polymers was extensively characterized and analyzed by AFM. Figure 7 shows the AFM images of HPAM with different concentrations. The HPAM solution exhibited a filamentous network structure. When the concentration was high (as shown in Fig. 7a), the fine filament structure overlapped and formed a thin lamellar structure. With decreasing concentration, the overlapped network structure gradually dispersed, forming the structure as shown in Fig. 7b. When the concentration was further reduced to $200 \mathrm{ppm}$ and $100 \mathrm{ppm}$ (Fig. 7c, d), respectively, most of the network nodes disappeared, the mesh structure increased, and the dense network structure became sparse.

HPAM solution with a concentration of $1000 \mathrm{ppm}$ was selected for the profile analysis, as shown in Fig. 8. The irregular network structure can be seen from the local enlarged diagram, and the skeleton of the network was not uniform. The height of the skeleton formed by HPAM was not homogeneous, more than 1-2 nm, as obvious from the three-dimensional stereogram. The AFM morphology analysis results showed that increasing the concentration of HPAM led to aggregation. Therefore, the aggregated molecular chains dispersed to form a network structure.

Figure 9 shows the micrograph of OR-P(AM/NaA/ AMPS) in aqueous solution, indicating the disordered network structure of OR-P(AM/NaA/AMPS) was more elongated, superimposed, and assembled on layers. With decreasing concentration, the degree of aggregation decreased, and the long filament structure was broken and gradually disappeared. However, when the concentration was reduced to $200 \mathrm{ppm}$, the polymer structure still showed the presence of interwoven polymer on the substrate.

The three-dimensional stereogram and height analysis diagram (as shown in Fig. 10) show that the height of the aggregate was mainly between 0.5 and $1 \mathrm{~nm}$, and a part of the multilayer aggregation reached $1.5 \mathrm{~nm}$. This result indicated that the molecular chain retained its aggregation state, at both low and high concentrations.

Figure 11 shows the AFM image of CR-P(AM/NaA/ AMPS) in aqueous solution. The structure is similar to that of OR-P(AM/NaA/AMPS). Both copolymers showed filamentary random interlacing nets, and the disordered network structure was slender, stacked together, and assembled. With decreasing concentration, the degree of aggregation decreased, and the long filament structure was broken and gradually disappeared. The interwoven polymer structure was still obtained when the concentration was reduced to $200 \mathrm{ppm}$. The height analysis (Fig. 10) showed that the height was between 0.5 and $1 \mathrm{~nm}$, which was same as that of OR-P(AM/NaA/AMPS).

The AFM characterization results showed that the network structures of OR-P(AM/NaA/AMPS) and CR-P(AM/ NaA/AMPS) (as shown in Fig. 12) can be elongated with increasing density of polymer solution. However, increasing polymer concentration from low to high resulted in aggregation, which caused the chains to disperse and form a network 

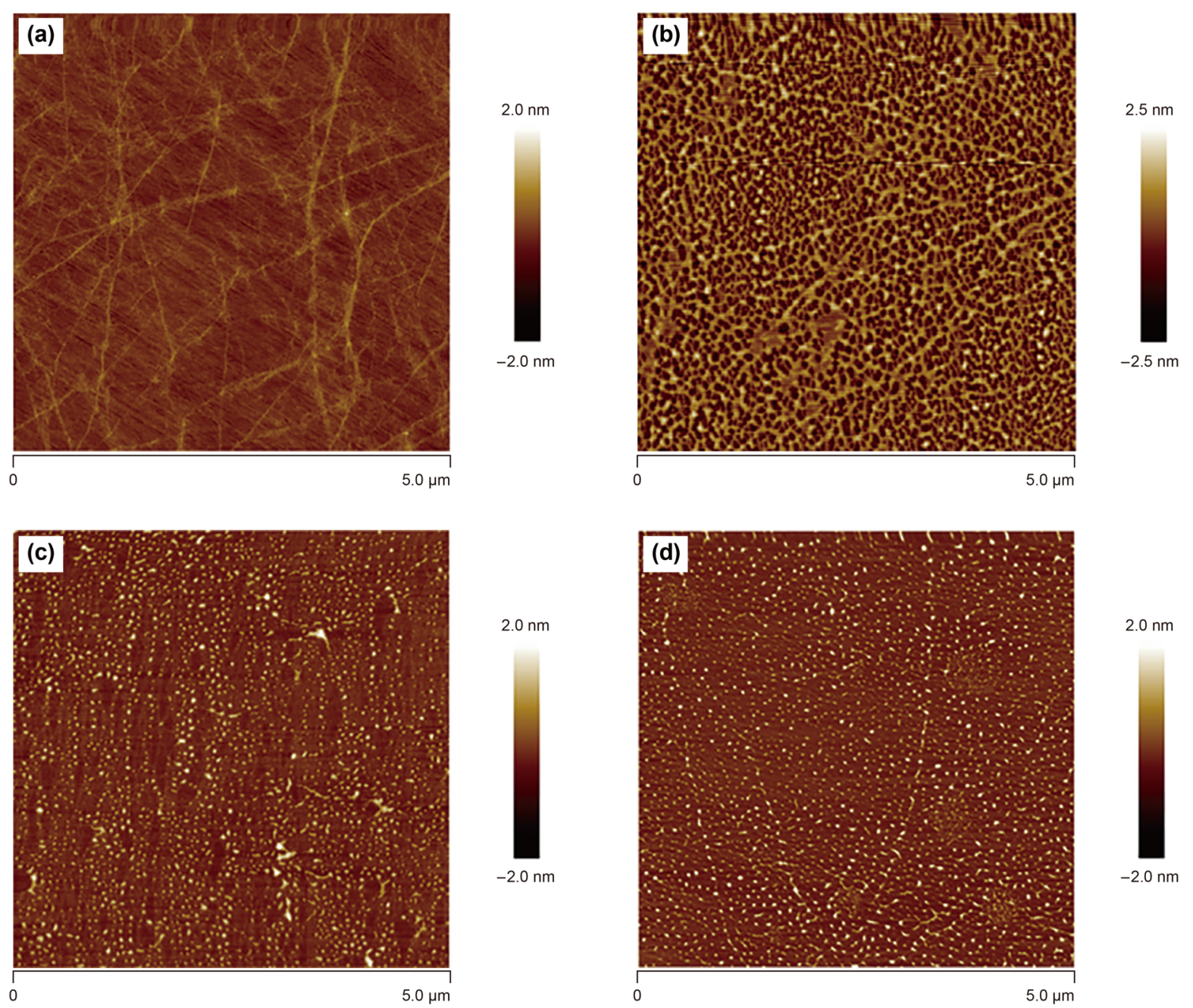

Fig. 7 AFM of HPAM; a-d is a height map, the concentration is 1000 ppm, 600 ppm, 200 ppm, 100 ppm
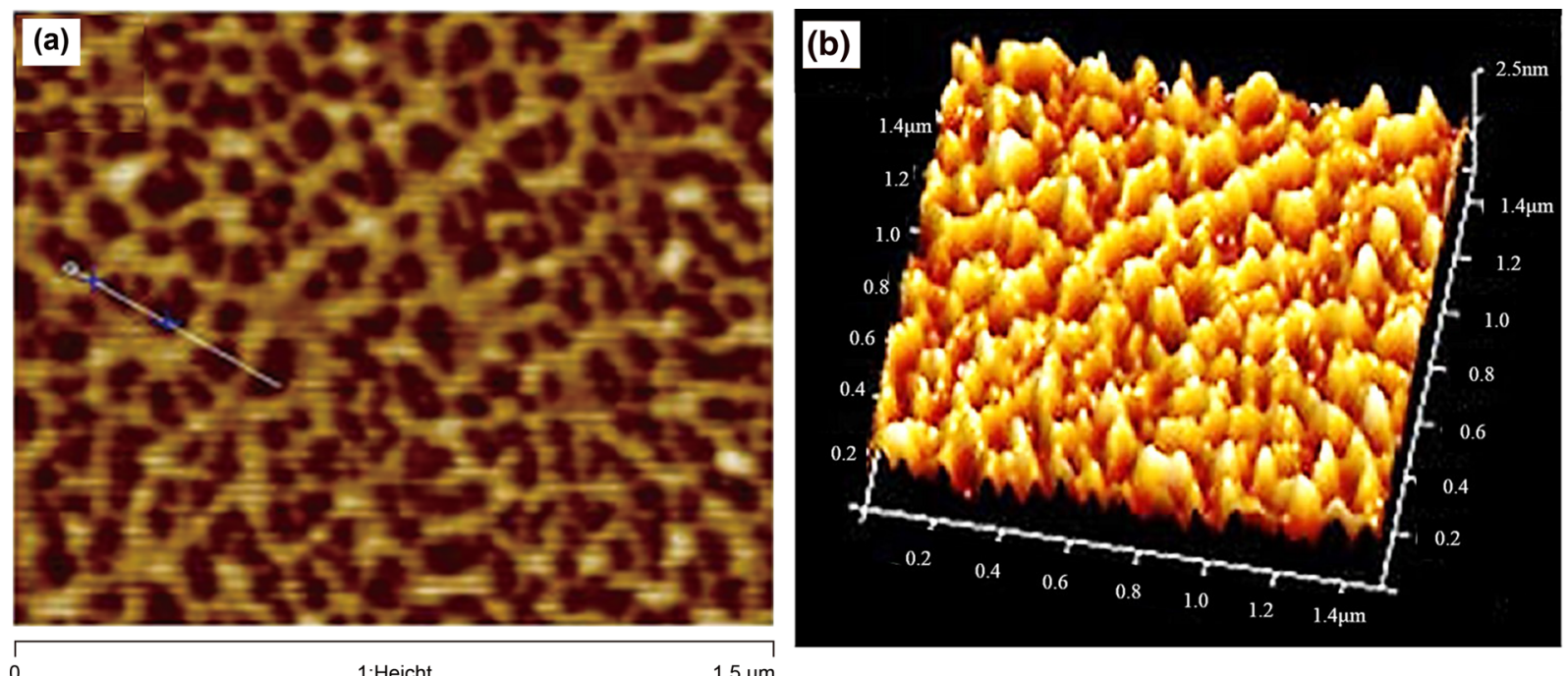

Fig. 8 Profile analysis of HPAM with a concentration of 1000 ppm; a local enlarged diagram; $\mathbf{b}$ three-dimensional stereogram 

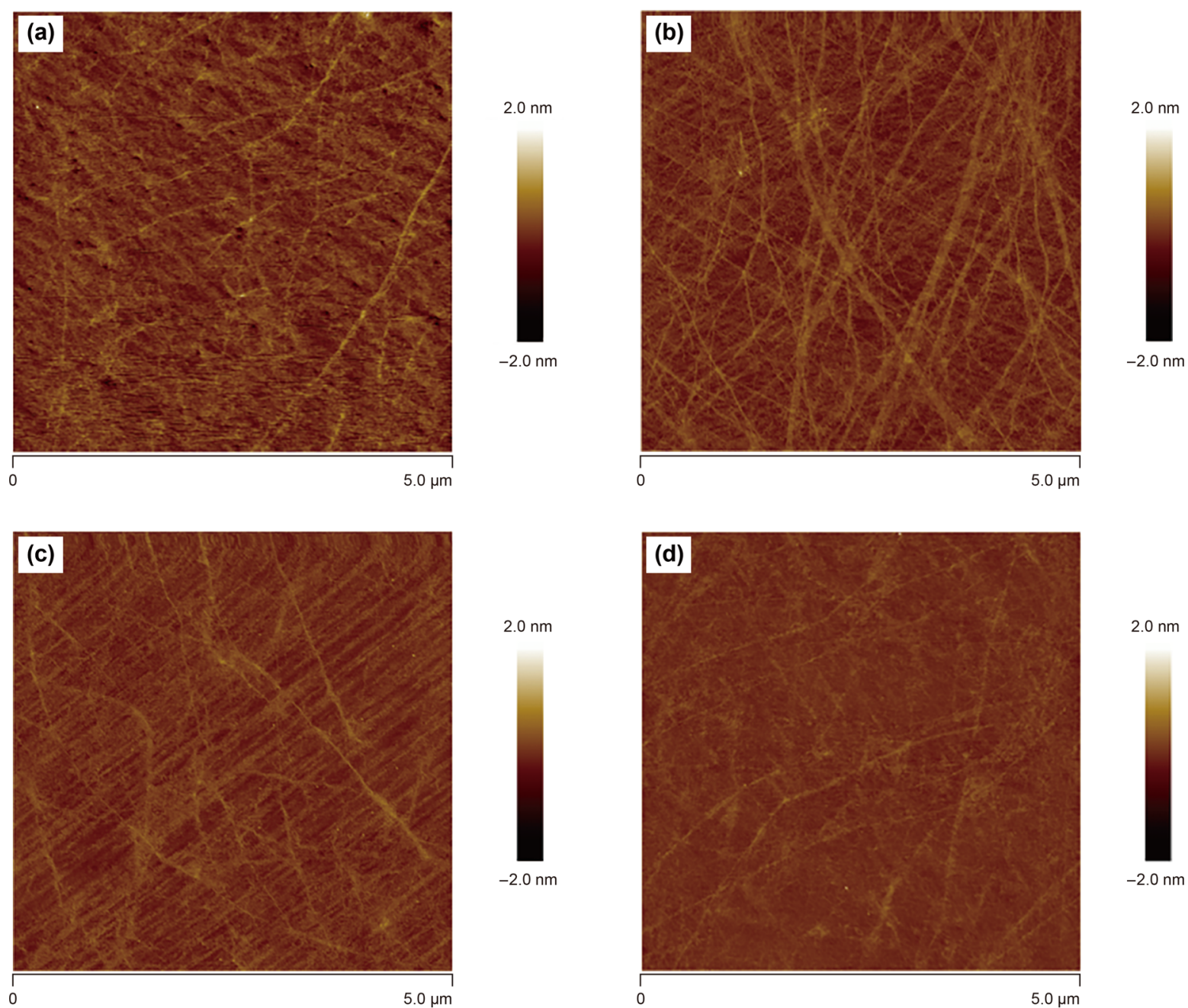

Fig. 9 AFM of OR-P(AM/NaA/AMPS); a-d is a height map, the concentration is 1000 ppm, 600 ppm, 200 ppm, 100 ppm
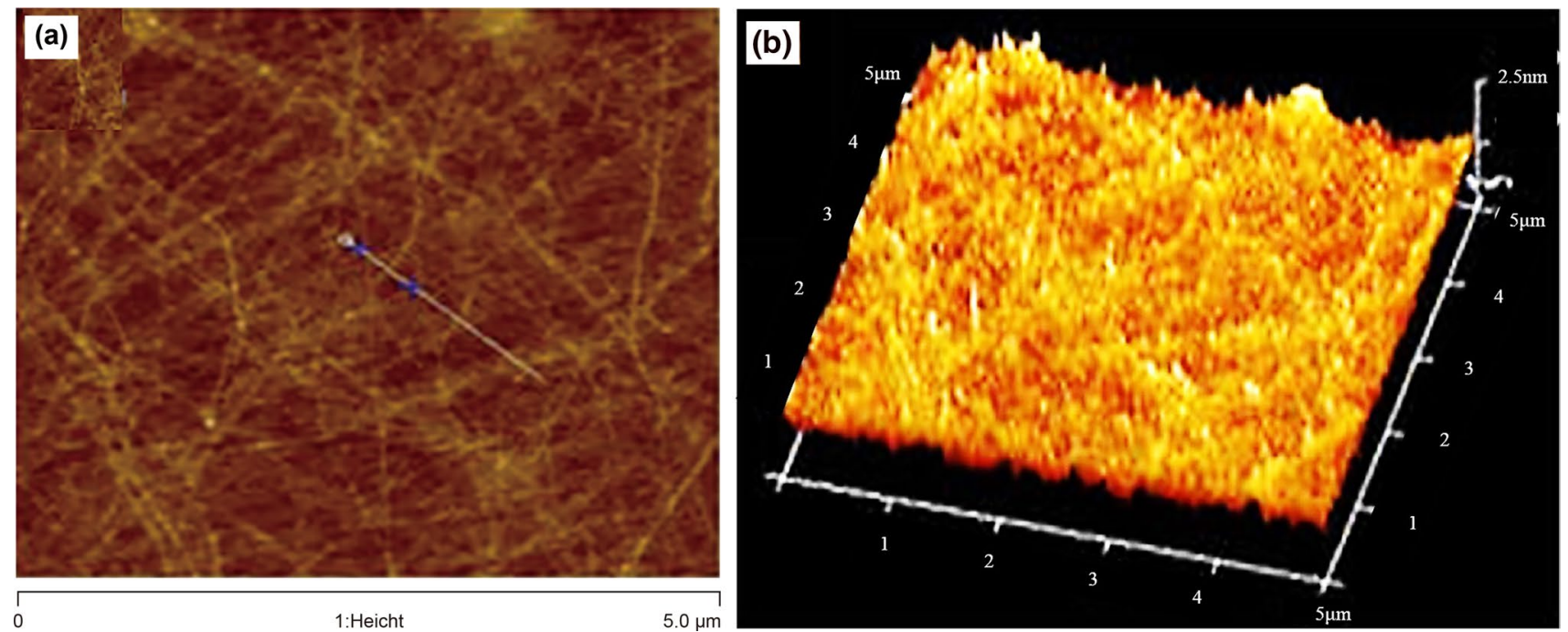

Fig. 10 Profile analysis of OR-P(AM/NaA/AMPS) with a concentration of $1000 \mathrm{ppm}$; a local enlarged diagram; b three-dimensional stereogram 

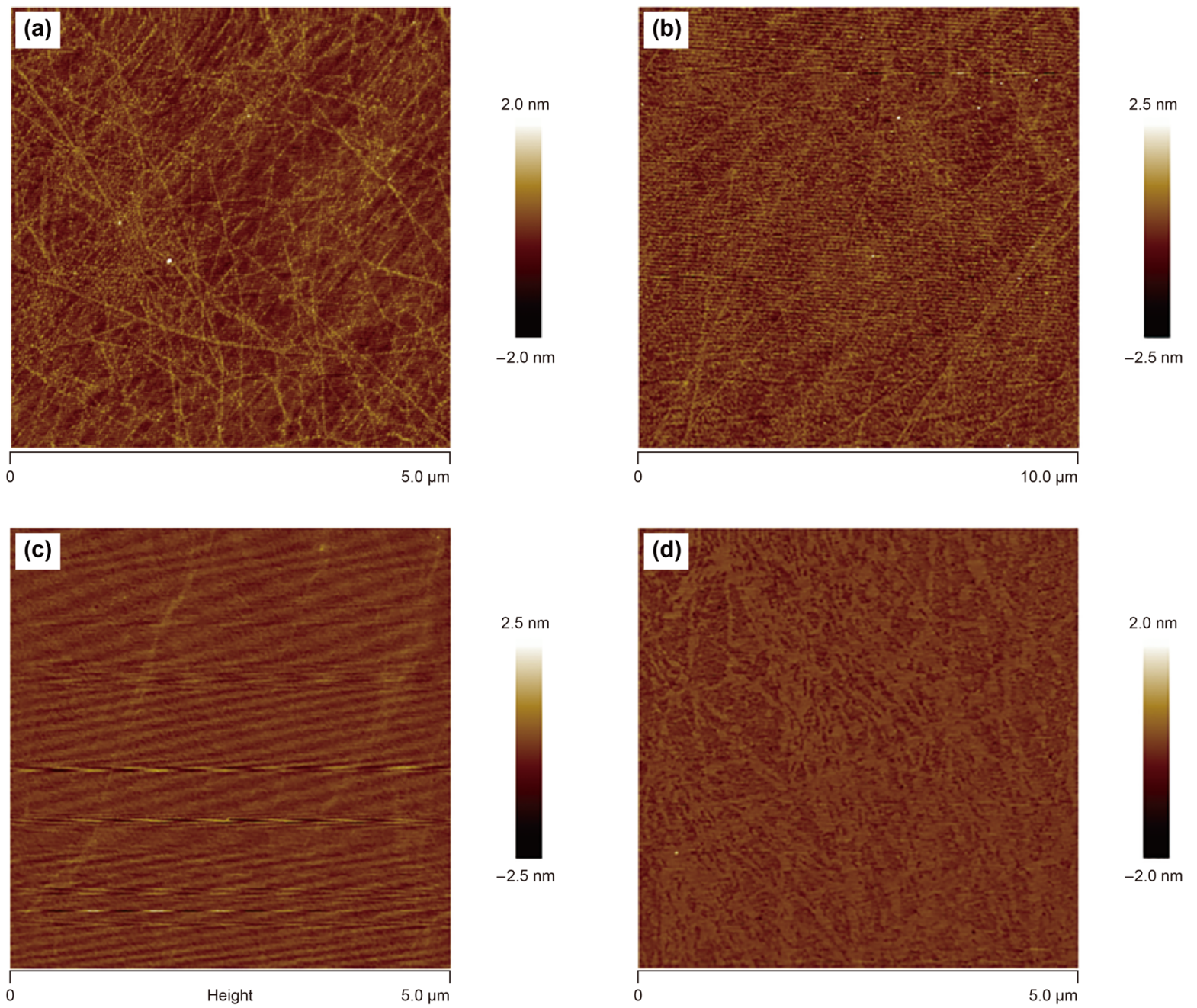

Fig. 11 AFM of CR-P(AM/NaA/AMPS); a-d is a height map, the concentration is 1000 ppm, 600 ppm, 200 ppm, 100 ppm
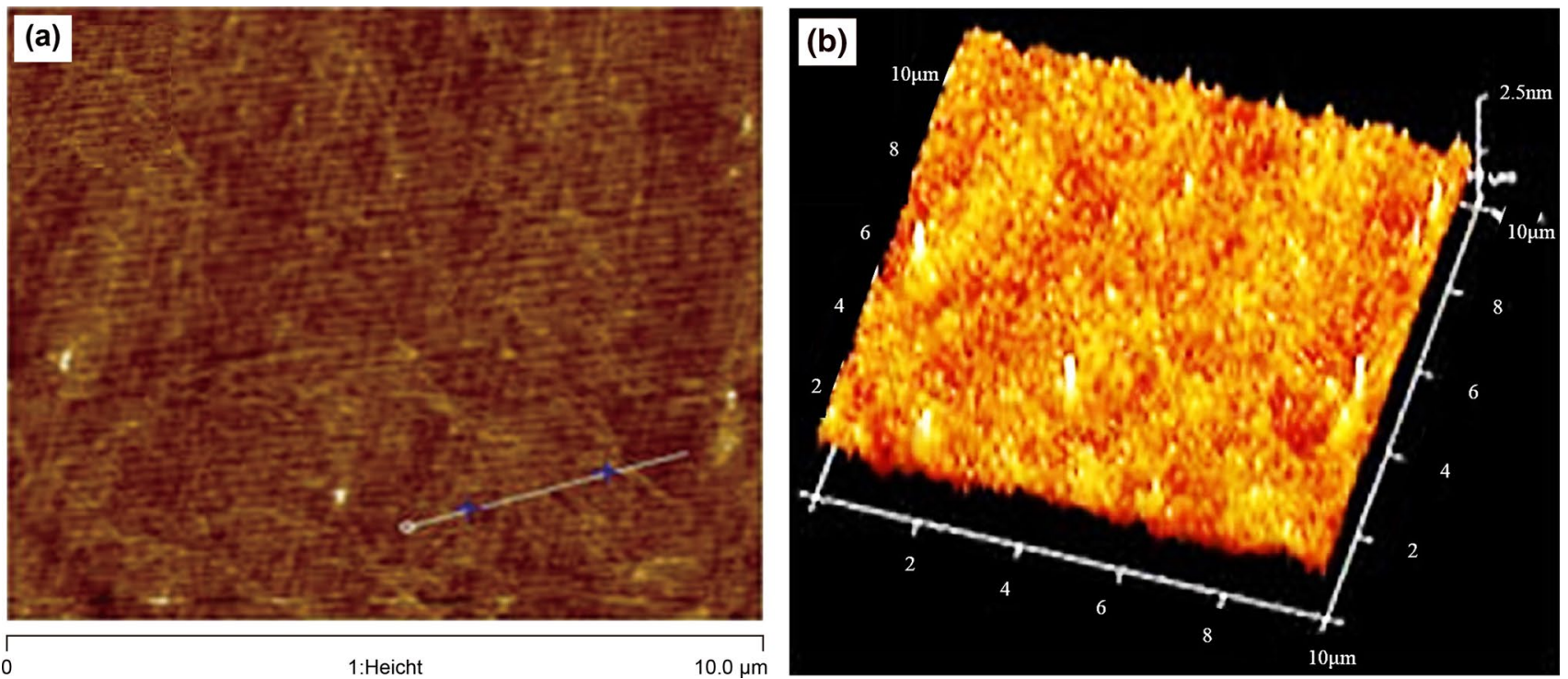

Fig. 12 Profile analysis of CR-P(AM/NaA/AMPS) with a concentration of $1000 \mathrm{ppm}$; a local enlarged diagram; $\mathbf{b}$ three-dimensional stereogram 
structure. This indicates that the two copolymer chains can form stronger aggregates than HPAM.

\section{Conclusion}

Two novel temperature-resistant and salt-tolerant copolymers (OR-P and CR-P) were successfully synthesized by the addition of AMPS monomer via OR and CR polymerization, respectively. The reaction conditions for their preparation were optimized, and the resulting copolymers were fully characterized. The results of TGA and freeze-etching SEM indicated that the temperature resistance OR-P(AM/ $\mathrm{NaA} / \mathrm{AMPS}$ ) and salt tolerance of CR-P(AM/NaA/AMPS) were enhanced by introducing functional AMPS monomer. The infrared spectra and elemental analyses provided strong evidence for grafting between AMPS and AM. The DLS results indicated that the two copolymers had higher hydrodynamic radius. CR-P(AM/NaA/AMPS) had the highest hydrodynamic radius, indicating its higher tackifying ability. The microcosmic aggregation morphologies of the three polymers were investigated by freeze-etching SEM and AFM. The results indicated that all three polymers formed net aggregates, while the net aggregates of OR-P and CR-P were stronger than that of HPAM. The network structure of CR-P(AM/NaA/AMPS) was more obvious than that of OR$\mathrm{P}(\mathrm{AM} / \mathrm{NaA} / \mathrm{AMPS})$ with increased salt tolerance, which was consistent with the DLS result.

Acknowledgements This research was supported by the National Science and Technology Major Project (No. 2016ZX05011-003), The Certificate of China Postdoctoral Science Foundation (No. 2016M592241).

Open Access This article is distributed under the terms of the Creative Commons Attribution 4.0 International License (http://creativeco mmons.org/licenses/by/4.0/), which permits unrestricted use, distribution, and reproduction in any medium, provided you give appropriate credit to the original author(s) and the source, provide a link to the Creative Commons license, and indicate if changes were made.

\section{References}

Bao M, Chen Q, Li Y, et al. Biodegradation of partially hydrolyzed polyacrylamide by bacteria isolated from production water after polymer flooding in an oil field. J Hazard Mater. 2010;184(13):105-10. https://doi.org/10.1016/j.jhazmat.2010.08.011.

Bara JE, Hatakeyama ES, Gabriel CJ, et al. Synthesis and light gas separations in cross-linked gemini room temperature ionic liquid polymer membranes. J Membr Sci. 2008;316(1-2):186-91. https ://doi.org/10.1016/j.memsci.2007.08.052.

Corlay P, Delamaide E, Petrole IFDU. Status and outlook of the polymer flooding process. J Petrol Technol. 1996;48(3):198-9. https ://doi.org/10.1115/1.2792698.
Ding C, Ju B, Zhang S. Temperature resistance and salt tolerance of starch derivatives containing sulfonate groups. Starch Stärke. 2014;66(3-4):369-75. https://doi.org/10.1002/star.201300127.

Feng YJ, Wang ZY, Li FS, et al. Study on solubility of hydrophobically modified polyacryamides. J Southwest Pet Inst. 2001;23(6):56-9. https://doi.org/10.3863/j.issn.1000-2634.2001.06.17 (in Chinese).

Gao C. Viscosity of partially hydrolyzed polyacrylamide under shearing and heat. J Pet Explor Prod Technol. 2013;3(3):203-6. https ://doi.org/10.1007/s13202-013-0051-4.

Gaynor SG, Wang JS, Matyjaszewski K. Controlled radical polymerization by degenerative transfer: effect of the structure of the transfer agent. Macromolecules. 1995;28(28):8051-6.

Guo J, Shi X, Yang Z, et al. Synthesis of temperature-resistant and salt-tolerant surfactant SDB-7 and its performance evaluation for Tahe Oilfield flooding (China). Pet Sci. 2014;11(4):584-9. https ://doi.org/10.1007/s12182-014-0375-9 (in Chinese).

$\mathrm{He} \mathrm{Y,} \mathrm{Xu} \mathrm{ZH,} \mathrm{Wu} \mathrm{F,} \mathrm{et} \mathrm{al.} \mathrm{Synthesis} \mathrm{and} \mathrm{characterization} \mathrm{of} \mathrm{a} \mathrm{novel}$ amphiphilic copolymer containing $\beta$-cyclodextrin. Colloid Polym Sci. 2014;292(7):1725-33. https://doi.org/10.1007/s0039 6-014-3235-7.

Hideharu M, Hiroshi O, Shuji N, et al. Xanthate-mediated controlled radical polymerization of $\mathrm{N}$-vinylcarbazole. Macromol Chem Phys. 2010;207(12):1005-17.

Hong CY, You YZ, Pan CY. Synthesis of water-soluble multiwalled carbon nanotubes with grafted temperature-responsive shells by surface RAFT polymerization. Chem Mater. 2005;17(9):2247-54. https://doi.org/10.1021/cm0480541.

Kafouris D, Themistou E, et al. Synthesis and characterization of star polymers and cross-linked star polymer model networks with cores based on an asymmetric, hydrolyzable dimethacrylate crosslinker. Chem Mater. 2006;18(1):119-43. https://doi.org/10.1021/ ma0513416.

Ladaviere C, Delair T, Domard A, et al. Studies of the thermal stability of maleic anhydride co-polymers in aqueous solution. Polym Degrad Stab. 1999;65(2):231-41. https://doi.org/10.1016/S0141 -3910(99)00009-9.

Liu X, Jiang W, Gou S, et al. Synthesis and evaluation of novel water-soluble copolymers based on acrylamide and modular $\beta$-cyclodextrin. Carbohydr Polym. 2013;96(1):47-56. https://doi. org/10.1016/j.carbpol.2013.03.053.

Ma J, Cui P, Zhao L, et al. Synthesis and solution behavior of hydrophobic association water-soluble polymers containing arylalkyl group. Eur Polym J. 2002;38(8):1627-33. https://doi.org/10.1016/ S0014-3057(02)00034-4.

Mao H, Qiu Z, Shen Z, et al. Hydrophobic associated polymer based silica nanoparticles composite with core-shell structure as a filtrate reducer for drilling fluid at utra-high temperature. J Pet Sci Eng. 2015;129:1-14. https://doi.org/10.1016/j.petrol.2015.03.003.

Sarsenbekuly B, Kang W, Fan H, et al. Study of salt tolerance and temperature resistance of a hydrophobically modified polyacrylamide based novel functional polymer for EOR. Colloids Surf A. 2017;514:91-7. https://doi.org/10.1016/j.colsurfa.2016.10.051.

Save M, Guillaneuf Y, Gilbert RG. Controlled radical polymerization in aqueous dispersed media. Cheminform. 2007;38(8):693-711.

Song H, Zhang SF, Ma XC, et al. Synthesis and application of starchgraft-poly(AM-co-AMPS) by using a complex initiation system of CS-APS. Carbohydr Polym. 2007;69(1):189-95. https://doi. org/10.1016/j.carbpol.2006.09.022.

Thomas S. Enhanced oil recovery-an overview. Oil Gas Sci Technol. 2007;63(1):9-19. https://doi.org/10.2516/ogst:2007060.

Wever DAZ, Picchioni F, Broekhuis AA. Polymers for enhanced oil recovery: a paradigm for structure-property relationship in aqueous solution. Prog Polym Sci. 2011;36(11):1558-628. https://doi. org/10.1016/j.progpolymsci.2011.05.006.

$\mathrm{Wu}$ YM, Zhang BQ, Wu T, et al. Properties of the forpolymer of N-vinylpyrrolidone with itaconic acid, acrylamide and 
2-acrylamido-2-methyl-1-propane sulfonic acid as a fluid-loss reducer for drilling fluid at high temperatures. Colloid Polym Sci. 2001;279(9):836-42. https://doi.org/10.1007/s003960100494.

Yang FY, Liu KY, Han SZ. Synthesis of super high molecular weight co-polymer of AM/AA/AMPS and its salt by inverse suspension polymerization. J Beijing Univ Chem Technol. 2003;30(2):5-9. https://doi.org/10.7688/j.issn.1000-1646.2013.02.07.

Ye ML, Han D, Shi LH. Studies on determination of molecular weight for ultrahigh molecular weight partially hydrolyzed polyacrylamide. J Appl Polym Sci. 2015;60(3):317-22. https://doi. org/10.1002/(SICI)1097-4628(19960418)60:33.0.CO;2-O.
Zhang XF, Zhang X, Dai H. Synthesis and solution behaviour of temperature sensitive water soluble polymers bearing alkylaryl hydrophobes. Plast Rubber Compos. 2007;36(7-8):365-9. https://doi. org/10.1179/174328907X237520.

Zhong C, Jiang L, Peng X. Synthesis and solution behavior of comblike terpolymers with poly(ethylene oxide) macromonomer. J Polym Sci Part A Polym Chem. 2010;48(5):1241-50. https://doi. org/10.1002/pola.23888. 